\title{
Prospects of essential oils in drug discovery
}

\begin{abstract}
Essential oils (EO), according to the International Standard Organization (ISO) defines essential oil as the product obtained from parts of plants by distillation with water steam and the product obtained by expression from pericarp of medicinal plant. The rapid growth and increase of the knowledge of identification, extraction, purification and characterization of essential oils in most local plant species is fast becoming an important aspect of the drug production process. Despite the complex nature of the essential oils in most plants in nature, it has significant impact in field of Medicine, Pharmacy and Aromatherapy. This work therefore reviews selected plants containing essential oils that are of importance to the drug discovery process. Issues concerning efficient screening of essential oils and implementation into drug discovery were discussed as well.
\end{abstract}

Volume 2 Issue I - 2017

\author{
Oludare Temitope Osuntokun, Adewale \\ Joseph Ogunleye \\ Department of Microbiology, Adekunle Ajasin University, Nigeria
}

Correspondence: Oludare Temitope Osuntokun, Department of Microbiology, Adekunle Ajasin University, PMB 00I, Akungba Akoko, Ondo state, Nigeria, Email osuntokun4m@yahoo.com, oludare.osuntokun@aaua.edu.ng

Received: November 25, 2016 | Published: February 02, 2017

\section{Introduction}

Essential oils (EO), or quinta essentia are complex mixtures of volatile secondary metabolites, found in plants naturally, which can only be extracted by hydro or steam distillation. The main constituents of essential oils are monoterpenes and sesquiterpenes, along with carbohydrates, alcohols, ethers, aldehydes, and ketones which are not oily to touch. They are responsible for the aroma and biological properties of medicinal plants. ${ }^{1}$ Bakali et al. ${ }^{2}$ described essential oils as 'the soul of plants', i.e. they form the odoriferous essence of plants. Essential oils are fragile and therefore handled with care because of its sensitive and volatile nature. Unlike, vegetable oils, they are isolated in small quantities that require special handling, care and packaging. The pharmaceutical importance of essential oils is relevant in virtually all forms of disease aetiology. Essential oils have been proved to demonstrate anti-microbial, anti-inflammatory, antioxidant, antitumorigenic and anti-carcinogenic properties as well as antibacterial, antiviral, antifungal and anti-parasitic properties. ${ }^{3}$ In this review, emphasis will be laid on the microbiological importance of essential oils in the development of novel antimicrobials and production of antibiotics.

One major problem with essential oils is in designing microbiological assays for the assessment of their effects on biological systems. The dispersion of the multivariate hydrophobic components of essential oils in the growth medium is a main problem in testing the activity of essential oils. In an attempt to solve this, essential oils are dissolved in organic solvents. However, some of these solvents are antiseptic in nature and they may interfere with the results of antimicrobial assays.

\section{Nomenclature of essential oils}

An essential oil is simply named according to the common name of its source plant. Plants such as ginger, lemon, cherry, almond, nutmeg, cypress, lavender tree etc. are named as ginger essential oil, lemon essential oil, almond essential oil, nutmeg essential oil, lavender essential oil (or sometimes oil of lavender) respectively. Another system of naming essential oils is the use of the generic name (from botanical name) of the source plant. Example includes the Spondias essential oil, Zingiber essential oil (Ginger), Citrus essential oil (Orange) etc.,. ${ }^{4}$

\section{Selected plants and their essential oil constituents}

Aspilia Africana (Pers.): Aspilia Africana (Pers.) is one of the most important sources of essential oils. It is an important member of the daisy family Asteraceae. It is a tropical tree, native to Africa, but now widely cultivated in Africa, especially in Nigeria and the Latin America. ${ }^{5}$ It is used in traditional African medicine for the treatment of various disorders such as wound dressings, pulmonary disorders, inflammation and Trichomonas infection. The chief constituents of A. Africana essential oil $(0.52 \% \mathrm{v} / \mathrm{w})^{6}$ are germacrene D $(28.4 \%)$, alpha-pinene (17.1\%), beta-pinene (4.1\%) beta-carophyllene $(7.5 \%)$, alpha-humulene (4.3), alpha-cubebene $(0.6 \%)$, and limonene $(1.1 \%)^{6}$ (for the full table see ref. 13). Aspilia essential oil is known to be inhibitory to a broad spectrum of microorganisms with an effective antibacterial activity against 12 common bacterial species including those from the genera Escherichia, Salmonella, Shigella, Staphylococcus, Pseudomonas and a host of others. ${ }^{\text {? }}$

Cinnamomum spp.: Specifically C. cassia and C. osmophloeum are good sources of essential oils. Cinnamomum spp are a group of hardwood trees of the Lauraceae family, in the order Laurales. ${ }^{8}$ Cinnamomum cassia (popularly known as cinnamon) is a much desired spice of choice throughout the world. Although the tree is native to South Asia and South America, its flavour is applied worldwide to spice up and preserve food. A growing body of evidence showed that essential oil from cinnamon (i.e. cinnamon essential oil) has antifungal and antibacterial activities. ${ }^{9}$ Hydro-distillation of cinnamon leaves revealed that $90 \%$ of the cinnamon essential oil contains cinnam-aldehyde while the remaining $10 \%$ is accounted for by eugenol, cinnamic acid and weitherhin. However, essential oil isolated from cinnamon tree bark showed that eugenol is the chief constituent. ${ }^{10}$ Cinnam-aldehyde alongside other volatile compounds of cinnamon essential oil such as eugenol showed $99.4 \%$ antimicrobial (both antifungal and antibacterial) properties. When compared with its decoction and infusion extracts, cinnamon essential oil was found to be greater by $29.2 \%$ and $47.2 \%$, respectively in terms of antimicrobial activity. ${ }^{11}$

Lipia multiflora moldenke: Lipia multifora Moldenke is a shrubby aromatic plant of the family Verbenaceae, which consists of up to 41 genera and 220 species. It is native to West Africa but it is now widely cultivated throughout tropical Africa, West and Central America. ${ }^{12}$ The volatile oils of L. multiflora have been extensively investigated for their medicinal properties. The bulk of the chemical components 
of Lipia essential oils are oxygenated monoterpenoids such as thymol, 1, 8-cineole and Eugenol. Basically, eugenols are natural antimicrobials, but eugenols from L. multiflora demonstrate a broad spectrum antimicrobial efficacy. The same applies to thymols, suggesting Lipia essential oils as leads of antimicrobials including antiviral, antibacterial and antifungal. In fact, there is an interesting synergy between eugenols and thymols that warrants further scientific investigations and applications.

Ocimum bassilicum linn: Ocimum bassilicum Linn. is an erect but small shrub (usually less than $1 \mathrm{~m}$ high), with a strong aromatic smell. The plant is endemic to West Africa and it can be found in gardens, around houses, forests and in the savannah. Ocimum bassilicum is used to inhibit epistaxis as well a febrifuge in traditional Yoruba medicine. To show that essential oils are responsible for its aromatic smell, leafy samples of $O$. basilicum and $O$. minimum were screened for essential oils comparatively. First, it was established that $88.1 \%$ and $74.4 \%$ of both extracts comprise essential oils. The investigation showed that methyl eugenol $(78.02 \%), \alpha$-cubebene $(6.17 \%)$, nerol $(0.83 \%)$ and $\varepsilon$-muurolene $(0.74 \%)$ are the most abundant. ${ }^{13}$ Microbiological screening of Ocimum essential oil infers that it has a broad spectrum of antibacterial activities, inhibiting the multidrug resistant Staphylococcus aureus, Staphylococcus epidemidis, Enterococci faecalis, Pseudomonas aeruginosa, Escherichia coli, Shigella dysentari$a e$, and the multidrug resistant Salmonella typhi.

Spondias mombin linn: Spondias spp are a group of tropical tress that grow in tropical countries of the world [14]. Spondias mombin is a tropical plant believed to have originated from the tropical Americas including the West Indies. Spondias mombin Linn belongs to the family Anacardiacae ${ }^{6}$ Spondias mombin is a very common tree among the Yoruba of South-Western Nigeria where it is used in Yoruba folk medicine. A careful but thorough screening for essential oils from Spondias mombin showed that it contains high levels of terpenes such as octadecane $(43.51 \%)$, tetracantane $(10.51 \%)$, hexatriacontane $(15.37 \%)$, $\beta$-caryophyllenes (delta-cadinine, alpha-murolene, alpha-gurjunene, 5 -isocedranol and-cadinene). ${ }^{14} \beta$-carophyllenes are the natural bicyclic sesquiterpenes which are responsible for the antibacterial, anti-inflammatory and the anti-tumour activities of the plant. ${ }^{14}$

\section{Essential oils in new drug discovery}

The use of medicinal plants and herbal products by some cultures has been long existing. ${ }^{15}$ Today, they are forming an integral aspect of the modern trend in new drug discovery. The role of essential oils in the discovery of new drugs cannot be overemphasized, especially in an era of antimicrobial resistance. With essential oils, one may suggest that certain abundant and effective constituents such as thymol, $\beta$-carophyllenes and eugenol as major highlights so far for development of potent antimicrobials. This is due to the fact that they possess novel chemistry and chemo types which could offer a remarkable opportunity for discovery of new drugs. So many antimicrobial resistant organisms including methicillin resistant staphylococcus aureus (MRSA) ${ }^{10}$ can be controlled using essential oils. Researchers are therefore encouraged to tailor drug design towards the use of essential oils (especially eugenols and thymols) to curb antimicrobial resistance. Other pharmaceutical potentials of essential oils include treatment of diseases such as neurodegenerative disease, heart diseases, pulmonary disorders and cancer.

Nevertheless, a lot of issues such as those concerning the pharmacological safety of essential oils, drug-herb interactions, long term efficacy, dosage formulation and ethical considerations still $^{1}$ need to be considered before the pharmaceutical clearance of essential oils into standard drugs. Just like antibiotics, the long term use of some essential oils could trigger wrong immune responses such as hypersensitivity, and inflammation. Extensive and intensive research on the toxicological and pharmacological effects of essential oils needs to be carried out before an essential oil product is termed 'Generally Regarded as Safe' (GRAS).

\section{Conclusion}

In the early parts of last year, the World Economic Forum (WEF) as reported in European Pharmaceutical Review, ${ }^{7}$ declared total war on antimicrobial resistance in the $21^{\text {st }}$ century. In response to this, new alternative to existing antibiotics and antifungal compounds must be sought. However, exploring the multivariate applications of essential oils such as creating drug synergies, and combinations may be a head way. From the review so far, it can be summed up that the essentiality of two main essential oils namely thymol and eugenol as well as some plant specific EOs such as cinnam-aldehyde are indispensable especially with the rise in antimicrobial resistance. These two constituents represent a common factor in virtually all plants used in aromatherapy. Understanding the interactions of these two oils and there long term effects in human body represent a giant stride in our battle against antimicrobial resistance.

\section{Acknowledgements}

None.

\section{Conflict of interest}

The author declares no conflict of interest.

\section{References}

1. Mendonça Filho RR. Bioactive Phytocompounds: New Approaches in the Phytosciences. In: Ahmad I, et al. editors. Modern Phytomedicine: Turning Medicinal Plants into Drugs. Germany: Wiley-Vch; 2016. p. $1-23$.

2. Bakkali F, Averbeck S, Averbeck D, et al. Biological effects of essential oils - A review. Food Chem Toxicol. 2008;46:446-475.

3. Chattopadhyay D. Ethnomedicinal antivirals: scope and opportunity. In: Ahmad I, et al. editors. Modern Phytomedicine: Turning Medicinal Plants into Drugs. Germany: Wiley-Vch; 2006. p. 313-335.

4. Directory of essential oils: Properties, Uses and Benefits alphabetized by botanical names. 2016 .

5. Flan G. International compositae alliance: Global compositae checklist (GCC). 2000.

6. Ogunwande IA, Eresanya O, Avoseh NO, et al. Chemical Composition of Essential Oils from Nigerian Plants. Der Chemica Sinica. 2012;3(1):279-286.

7. Lu SM, Chen JX, Chen ZL. A study on the in vitro bacteriostatic action of eugenol. Shi Pin Ke Xue. 2008;29(9):122-124.

8. Nostro A. Activity of Plant Extracts and Plant-Derived Compounds against Drug-Resistant Microorganisms. In: Ahmad I, Aqil F, et al. editors. Modern Phytomedicine: Turning Medicinal Plants into Drugs. Germany: Wiley-Vch; 2006. p. 199-226.

9. Fabio AA, Corona E, Forte, P Quaglio. Inhibitory activity of spices and essential oils on psychrotrophic bacteria. Microbiol. 2003;26(1):115120 . 
10. Ahmad N, Alan M, Ahmad I, Owais M. Use of a liposomal delivery system for herbal-based therapeutics (with a focus on clove oil). In: Ahmad I, et al. editors. Modern Phytomedicine: Turning Medicinal Plants into Drugs. Germany: Wiley-Vch; 2006. p. 357-366.

11. Chaudhry NMA, Tariq P. Anti-microbial activity of cinnamomum cassia against diverse microbial flora with its nutritional and medicinal impacts. Pak J Bot. 2006;38(1):169-174.

12. Omoregie EH, Folashade KO. Essential oil of lipia multiflora moldenke: a review. Journal of Applied Pharmaceutical Science. 2012;2(1):15-23.
13. Ozcan M, Chalchat J. Essential Oil Composition of Ocimum basilicum $L$ and Ocimum minimum $L$ in Turkey. Czech J Food Sci. 2002;20(6):223-228.

14. Ayoka AO, Akomolafe RO, Akinsomisoye OS, et al. Medicinal and economic value of spondias mombin. African Journal of Biomedical Research. 2008;11:129-136.

15. Halberstein RA. Medicinal plants: Historical and cross-cultural usage patterns. Ann Epidemiol. 2005;15(9):686-699.

16. European pharmaceutical review. News: pharma calls for collective action to combat antimicrobial resistance. 2016. 